\title{
The Hyperlipidemia Caused by Overuse of Glucocorticoid after Liver Transplantation and the Immune Adjustment Strategy
}

\author{
Xueqin Meng, ${ }^{1,2,3}$ Xinhua Chen, ${ }^{1,2,3}$ Liming $W u,{ }^{1,2,3}$ and Shusen Zheng ${ }^{1,2,3}$ \\ ${ }^{1}$ Collaborative Innovation Center for Diagnosis and Treatment of Infectious Diseases, The First Affiliated Hospital, \\ Zhejiang University, Hangzhou 310003, China \\ ${ }^{2}$ Key Laboratory of Combined Multi-Organ Transplantation, Ministry of Public Health, The First Affiliated Hospital, \\ Zhejiang University, Hangzhou 310003, China \\ ${ }^{3}$ The Department of Hepatobiliary and Pancreatic Surgery, The First Affiliated Hospital, Zhejiang University, \\ Hangzhou 310003, China
}

Correspondence should be addressed to Shusen Zheng; shusenzheng@zju.edu.cn

Received 29 July 2016; Revised 25 September 2016; Accepted 28 December 2016; Published 17 January 2017

Academic Editor: Andréia M. Cardoso

Copyright (C) 2017 Xueqin Meng et al. This is an open access article distributed under the Creative Commons Attribution License, which permits unrestricted use, distribution, and reproduction in any medium, provided the original work is properly cited.

\begin{abstract}
The overuse of glucocorticoid may cause the metabolic disorders affecting the long term outcome of liver transplantation. This study aims to investigate the immune adjustment strategy by decreasing use of glucocorticoid after liver transplantation. The follow-up study was carried out on liver function and lipid metabolism. This study included adult recipients of liver transplantation. There were 3 groups according to their use of glucocorticoid: long term ( $>3$ months, $n=18)$, short term ( $<3$ months, $n=20)$, and control group (no use of glucocorticoid, radical hepatic resection, $n=22$ ). The laboratory results of liver function (AST/ALT ratio) and serum lipid were compared 6 months after liver transplantation. AST/ALT ratio, the marker of liver function, showed no significant difference between long and short term group $(P>0.05)$. The acute rejection had no significant difference between short and long term groups, while TG, HDL, LDL, and glucose showed significant change in the long term group $(P<0.05)$. At 6 months after liver transplantation, the long term group showed higher metabolic disorders $(P<0.05)$. The proper immune adjustment strategy should be made to avoid overuse of glucocorticoid. It can decrease hyperlipidemia and other metabolic disorders after liver transplantation without increasing the acute rejection or liver function damage.
\end{abstract}

\section{Introduction}

After liver transplantation, the overuse of glucocorticoid will cause glucocorticoid-related complications, such as newonset diabetes, hypertension, osteoporosis, growth retardation, and hyperlipidemia [1]. With the application of new potent immune suppress agents, acute rejection after liver transplantation is not the main factor affecting the prognosis [2]. After raising the Hangzhou criteria, outcomes of liver transplant in our center continued to improve in recent decade. The liver transplantation recipients in $1-, 3-$, and 5-year overall survival rate achieved $90.41 \%$, $78.04 \%$, and $72.45 \%$ [3]. The allograft-related perisurgery complications are decreasing. Prevention of hyperlipidemia and other metabolic disorders can decrease cardiovascular complications and improve the long term outcomes. Liver transplant is considered a coronary heart disease high risk based on the National Cholesterol Education Program Expert Panel on Detection, Evaluation, and Treatment of High Blood Cholesterol in Adults [4]. The study aims to compare the different use of glucocorticoid and the impact on liver function and hyperlipidemia after liver transplantation.

\section{Materials and Methods}

2.1. Patients. End-stage liver cirrhosis patients who had liver transplantation were followed up. Liver disease pathology was confirmed by laboratory examinations and liver biopsies. The primary disease, surgery procedure, gender, and age in different groups were matched. The study was approved by the Ethics Committee of the First Hospital of Zhejiang University. 
TABLE 1: The baseline and characteristics of patients.

\begin{tabular}{lccc}
\hline & Control without glucocorticoid & Short term use of glucocorticoid & Long term use of glucocorticoid \\
\hline Number of patients & 22 & 20 & 18 \\
Time of glucocorticoid & 0 & $<3$ months & $>3$ months \\
Gender (male/female) & $10 / 12$ & $11 / 10$ & $10 / 8$ \\
Recipient age & $52 \pm 8.3$ & $53 \pm 7.4$ & $48 \pm 5.1$ \\
MELD score & $16.3 \pm 4.1$ & $24.9 \pm 7.3$ & $22.9 \pm 6.7$ \\
Surgery & Hepatic resection & Orthotopic liver transplantation & Orthotopic liver transplantation \\
CSA & No & Yes & Yes \\
BMI & $24 \pm 3.4$ & $26 \pm 1.4$ & $28 \pm 1.1$ \\
Basic diseases & Angioma & Liver cirrhosis & Liver cirrhosis \\
\hline
\end{tabular}

The details were present in Table 1. The adrenocorticotropic hormone (ACTH) has been tested and Cushing's syndrome has been excluded.

We follow up the adult liver transplantation recipients with the matched disease and surgeries. There were 3 groups according to their use of glucocorticoid: long term ( $>3$ months, $n=18)$, short term ( $<3$ months, $n=20)$, and control group (no use of glucocorticoid, radical hepatic resection, and $n=22$ ). The laboratory results of liver function, serum lipid, and metabolic disorder occurrence were compared at 6 months after LT.

2.2. Immunosuppression Strategy. All transplantation recipients received immunosuppressants. The immunosuppressive medications (cyclosporine/corticosteroid) were in early stage after liver transplantation. We tried to cut off the time of corticosteroids within 3 months. $1000 \mathrm{mg}$ prednisolone on the first day and $20 \mathrm{mg}$ tapered to zero within the first 3 months.

2.3. Follow-Up Study. Adult recipients of liver transplantation were followed up according to the time of taking glucocorticoid: long term use of glucocorticoid ( $>3$ month, $n=18$ ), short term use of glucocorticoid ( $<3$ month, $n=20$ ), and control group (no use of glucocorticoid, radical hepatic resection, and $n=22$ ).

2.4. Laboratory Tests. Laboratory results of liver function and metabolic function 6 months after LT were compared. Blood samples following $12 \mathrm{~h}$ fasting and $2 \mathrm{~h}$ after dinner were drawn from vein for adrenocorticotropic hormone (ACTH), cortisol, glucose, and serum lipids (total cholesterol, LDL, HDL, and triglyceride). ACTH was tested by electrochemiluminescence Elecsys ACTH immunoassay, ECLIIA, (Roche Diagnostics, Mannheim, Germany) on E170 Model. Cortisol was tested by a chemiluminescence assay (Bayer, Shanghai, China) on the ACS180 SE Model. The biochemistry exams were done by automated chemistry analyzer (Hitachi, Tokyo, Japan) on Hitachi 7600-110 Model. The liver function and lipids were tested by BECKMAN COULTER analyzer (Beckman Coulter Diagnostics, CA, USA) on AU5811 Model.

We measured the new onset diabetes according to the American Diabetes Association/World Health Organization guidelines; new onset DM was defined as patient used to have

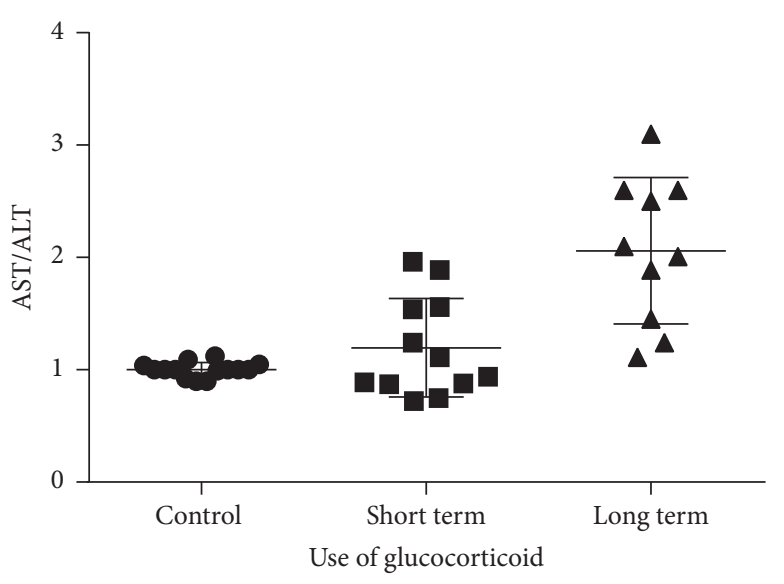

FIgure 1: The AST/ALT ratio in patients taking short term, long term, and no glucocorticoid. Levels of AST/ALT ratio were significantly higher $(P<0.05)$ in patients who took glucocorticoid no matter long term or short term compared with control. There were no significant difference of AST between short or long term groups. Data are expressed in AST/ALT which is a marker to reflect liver function damage.

a normal fasting blood glucose, but after liver transplantation, he/she has a fasting blood glucose of $\geq 7.00 \mathrm{mmol} / \mathrm{L}(1.26 \mathrm{~g} / \mathrm{L})$ confirmed on at least 2 occasions or current treatment with an oral antidiabetic drug or insulin. The pathological slides from the biopsy were parallel to the liver function results.

2.5. Statistical Analysis. Data were expressed as mean \pm SD. For statistical comparison of values, Student's $t$-test was used by software SPSS 17.0. $P$ values less than 0.05 were deemed to indicate statistical significance.

\section{Results}

3.1. AST/ALT Ratio Significantly Changed in Patients Who Took Glucocorticoid. The 3 groups of patients who took glucocorticoid at different times after liver surgery were shown in Figure 1. There was no acute rejection in recipients, indicating that the use of glucocorticoid helped the transplanted liver to work properly and, as a results, patients survived. AST/ALT ratio had no significant difference between short or long term 
TABLE 2: The complications in patients with use of glucocorticoid after liver transplantation.

\begin{tabular}{lccccc}
\hline & Infection & Wound nonhealing & New onset diabetes & Hypertension & Acute rejection \\
\hline Short term & 3 & 2 & 3 & 2 & 9 \\
Long term & 10 & 7 & 11 & 5.553217 & 3 \\
$\chi^{2}$ & 5.238479 & 6.788542 & 5.553217 & 0.018447 & 0.015993 \\
$P$ & 0.022093 & 0.009174 & 0.018447 & 0.899364 \\
\hline
\end{tabular}

TABLE 3: The baseline and characteristics of patients.

\begin{tabular}{|c|c|c|c|}
\hline & $\begin{array}{l}\text { Control without } \\
\text { glucocorticoid }\end{array}$ & $\begin{array}{l}\text { Short term use of } \\
\text { glucocorticoid }\end{array}$ & $\begin{array}{l}\text { Long term use of } \\
\text { glucocorticoid }\end{array}$ \\
\hline Number of patients & 22 & 20 & 18 \\
\hline Time of glucocorticoid & 0 & $<3$ months & $>3$ months \\
\hline Glucose fasting (mmol/L) & $4.9 \pm 1.3$ & $5.1 \pm 0.7$ & $5.3 \pm 1.1$ \\
\hline Glucose $2 \mathrm{~h}$ after dinner $(\mathrm{mmol} / \mathrm{L})$ & $6.9 \pm 0.7$ & $7.1 \pm 0.7$ & $9.3 \pm 2.6^{*}$ \\
\hline ACTH 8 am (pmol/L) & $6.5 \pm 0.4$ & $7.2 \pm 0.7$ & $7.9 \pm 0.3$ \\
\hline HDL (high density lipoprotein, mmol/L) & $1.49 \pm 0.31$ & $1.29 \pm 0.24$ & $0.99 \pm 0.21^{*}$ \\
\hline LDL (low density lipoprotein, mmol/L) & $2.29 \pm 0.81$ & $2.71 \pm 0.53$ & $3.09 \pm 0.74^{*}$ \\
\hline TC (total cholesterol, mmol/L) & $5.29 \pm 0.37$ & $5.61 \pm 0.21$ & $5.78 \pm 0.52$ \\
\hline
\end{tabular}

${ }^{*} P<0.05$ versus control group.

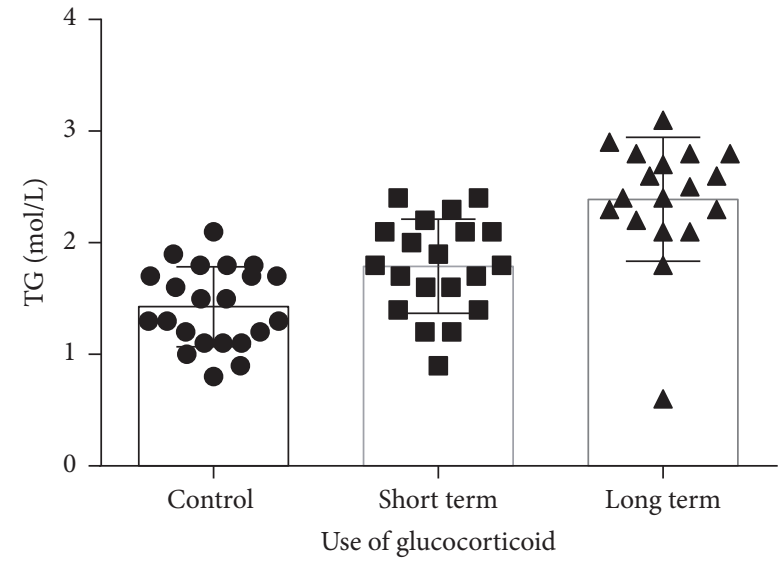

FIgure 2: The TG levels in patients taking short term, long term, and no glucocorticoid. The quantitative evaluation of TG was analyzed and expressed in mol/L. TG significantly increased in patients who took different time of glucocorticoid compared with the control group. When comparing short term and long term use of glucocorticoid, short term group significantly decreased versus long term group $(P<0.05)$.

groups, which indicates that the short use of glucocorticoid less than 3 months can achieve the same immune effect as long term use of glucocorticoid longer than 3 months.

\subsection{Cutting off the Overuse of Glucocorticoid Can Decrease}

$T G$. The quantitative evaluation of serum lipids was analyzed (Figure 2). TG significantly increased in patients who took different time of glucocorticoid compared with the control group $(P<0.05)$, indicating that the use of glucocorticoid can affect the lipid metabolism and lead to hyperlipidemia.
When comparing short term and long term use of glucocorticoid, short term group significantly had decreased TG versus long term group $(P<0.05)$, indicating that cutting off the overuse of glucocorticoid can effectively decrease TG.

3.3. The Acute Rejection Occurrence. With development of surgery skill and organ protection technology, acute rejection becomes less common. All episodes of acute rejection in this study occurred in the first 6 weeks in recipients with a donor mismatches (Table 2). The prevalence of acute rejection was $10 \%$ in short term group versus $17 \%$ in long term group $(P=$ 0.899364). Clinical symptoms were not typical. Laboratory tests on liver function were, for example, elevations AST (Figure 1). The diagnosis of acute rejection was confirmed by needle biopsy. The histopathological findings in reports from Department of Pathology showed that acute rejection includes predominantly mononuclear portal inflammation; subendothelial inflammation of portal and hepatic veins; and bile duct inflammation and damage. The short and long term groups had no significant difference in liver damage such as duct damages or unequivocal endotheliitis except some inflammatory infiltration in long term group. There were no visible steatosis cells in any groups (Figure 3 ).

3.4. The Metabolic Disorder Occurrence. Features of the metabolic disorders were found in patients 6 months after transplantation (Tables 2 and 3). New onset diabetes rose from $15 \%$ in short term group to $61 \%$ in long term group $(P=0.009174$, Table 2$)$; hypertension increased from $10 \%$ in short term group to $50 \%$ in long term group $(P=0.018447$, Table 2). The long term group had significantly reduced HDL and increased LDL and $2 \mathrm{~h}$ after dinner blood glucose (Table 3). 

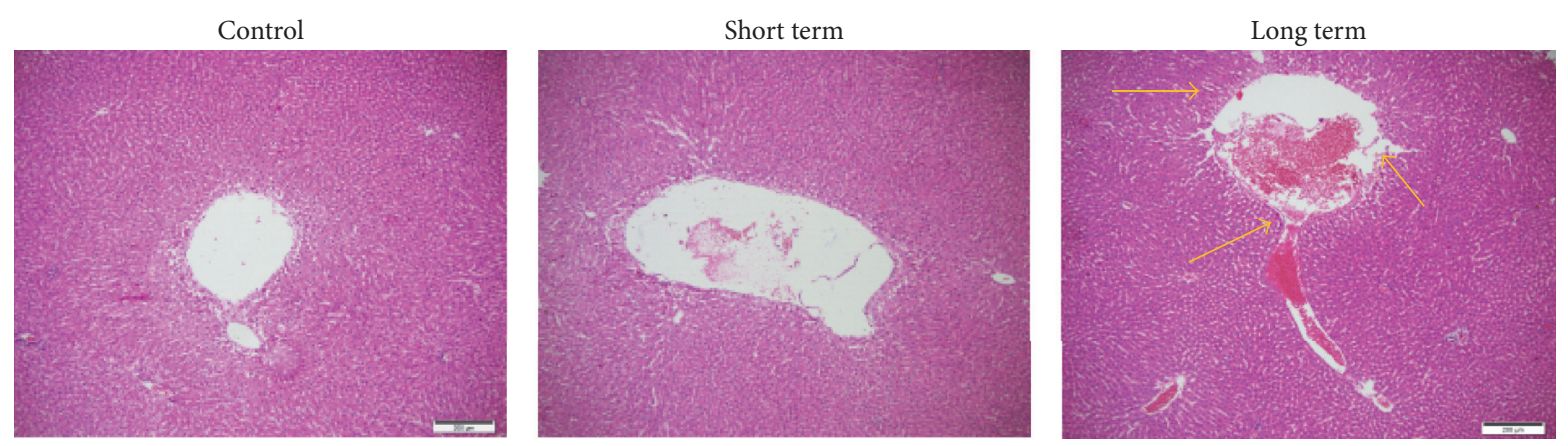

FIgURE 3: The histopathological results. The biopsy from patients in short term, long term, and control groups was shown. The short term indicates no significant difference from the control group while the long term group had sporadic inflammatory cells infiltration (the arrow indicates the lymphocytes). There was no obvious steatosis in any groups.

\subsection{The Occurrence of Glucocorticoid-Related Side Effects.} Occurrence of glucocorticoid-related side effects such as infection and poor wound healing was found in patients followed up $>6$ months after transplantation (Table 2). The prevalence of infection rises from $15 \%$ in short term group to $56 \%$ in long term group $(P=0.022093)$. Poor wound healing increased from $10 \%$ in short term group to $39 \%$ in long term group $(P=0.087380)$. The reinfection with hepatitis $\mathrm{B}$ and the infection with CMV have been excluded.

\section{Discussion}

Lipid metabolic disorders are becoming more common after liver transplantation which affect the long term morbidity and mortality as well as life quality. Some patients do have the metabolic preconditions such as overweight, diabetes, and cholestatic liver disease. The new onset of metabolic disorders is often caused by the use of immunosuppressant medications such as cyclosporine, tacrolimus, and glucocorticoid which attack the insulin signaling pathway by changing adipose enzymes [5].

We monitor patients' liver function and lipid levels 6 months after transplant. The results indicate that avoiding overuse of glucocorticoid can significantly decrease the risk of hyperlipidemia in liver transplant recipients.

It has been well known that glucocorticoid inhibits immune function. The metabolic side effects have been noticed recently such as diabetes, hypertension, and hyperlipidemia [6].

Because acute rejection after liver transplantation generally occurred 2 weeks after liver transplantation, we suggest early glucocorticoid withdrawal scheme to ensure a lower rejection and minimize the amount of glucocorticoid. Our clinical data suggest that the minimized use of glucocorticoid did not increase the incidence of rejection but decreased postoperative infection and poor wound healing. Quite different from some liver transplantation centers whose primary liver diseases of liver transplantation are alcoholic cirrhosis, HCV cirrhosis, and autoimmune liver disease, our center has more than $90 \%$ of liver transplant recipients for HBVrelated diseases, with about $40 \%$ of hepatocellular carcinoma. Overuse of glucocorticoid after liver transplantation for more than 1 year also causes the cancer and HBV recurrence $[7,8]$.

The blood glucose changes caused by the long term use of steroid are different from type 1 and type 2 diabetes in terms of etiology, clinical features, and treatment strategies. One unique finding is that the glucocorticoid-induced blood glucose changes on fasting and randomly were different. Because glucocorticoids were administered at 8:00 am, these patients' fasting glucose was not affected. But the $2 \mathrm{~h}$ blood glucose after dinner significantly increased compared to control group.

Steroids have been used for the purpose of preventing rejection. However, steroid-related metabolic disorders, such as hypertension, diabetes mellitus, hyperlipidemia, and obesity, are causing concerns for its long term use. Hyperlipidemia significantly increases cardiovascular events. In addition, the hyperlipidemia caused atherosclerosis, which can reduce liver perfusion, resulting in deterioration of liver function. Hyperlipidemia also participates in the chronic graft rejection similar to the proliferate vascular disease.

Our result demonstrated that a short term use of steroid ( $<3$ months) was safe in patients undergoing LT for HBVrelated HCC. Liver function recovered significantly better than those of long term use of steroid ( $>3$ months).

The patients who had liver transplantation for their autoimmune hepatitis do have difficulties stopping steroid because the high chance of immune hepatitis recurrence. There are a few patients who need to use steroid because of rejection. When the acute rejection occurs, high dose steroid is still the first line medicine. Other than the occasions mentioned above, the majority of adult recipients can stop steroid safely within 3 months. Previous study [9] concluded that steroid tapering to $5 \mathrm{mg}$ /day does not lead to graft loss and may decrease the incidence and severity of late metabolic complications. When there is no immune hepatitis nor acute rejection, the steroid can be discontinued $14 \mathrm{~d}$ after liver transplantation when immunosuppressive therapy based on FKS06+ MMF/CsA was still maintained [10, 11].

\section{Conclusion}

Glucocorticoid after liver transplantation is one of the most commonly used immunosuppressants, playing a very 
important role in the prevention and treatment of acute rejection. However, long term use of glucocorticoid has many side effects. Early withdraw within 3 months can decrease the risk of metabolic disorders.

\section{Competing Interests}

All the authors declare that they have no potential conflict of interests.

\section{Authors' Contributions}

Shusen Zheng and Liming Wu conceived and designed the experiments; Xueqin Meng, Xinhua Chen, and Liming $\mathrm{Wu}$ collected samples; Xueqin Meng and Xinhua Chen performed the experiment and analyzed data; and Xueqin Meng and Xinhua Chen wrote the manuscript and contributed equally to this paper.

\section{Acknowledgments}

The research is supported by National Natural Science Foundation of China $(81372425,81401319,81421062$, and 91542205), Xinjiang Cooperation project (2014KL002), and Zhejiang project (nos. 2013T301-15 and 2016C33145).

\section{References}

[1] Ž. Reiner, "Management of patients with familial hypercholesterolaemia," Nature Reviews Cardiology, vol. 12, no. 10, pp. 565575, 2015.

[2] X. Xu, D. Lu, Q. Ling et al., "Liver transplantation for hepatocellular carcinoma beyond the Milan criteria," Gut, vol. 65, no. 6, pp. 1035-1041, 2016.

[3] Z. Hu, J. Zhou, Z. Li et al., "Salvage liver transplantation for recurrent hepatocellular carcinoma after liver resection: retrospective study of the Milan and Hangzhou criteria," PLoS ONE, vol. 9, no. 1, Article ID e87222, 2014.

[4] A. Ayhan, C. Araz, O. Komurcu, S. Kaplan, A. Torgay, and M. Haberal, "The evaluation of hemodynamic changes during the reperfusion phase in adult living donor liver transplantations: the role of cardiovascular problems," Transplantation Proceedings, vol. 47, no. 4, pp. 1199-1203, 2015.

[5] C. Huang, P. Chiu, H. Liu et al., "Clinical observations and treatment of pediatric homozygous familial hypercholesterolemia due to a low-density lipoprotein receptor defect," Journal of Clinical Lipidology, vol. 9, no. 2, pp. 234-240, 2015.

[6] S. Gebhardt, M. Jara, M. Malinowski et al., "Risk factors of metabolic disorders after liver transplantation: an analysis of data from fasted patients," Transplantation, vol. 99, no. 6, pp. 1243-1249, 2015.

[7] K. Nikkilä, F. Åberg, and H. Isoniemi, “Transmission of LDLR mutation from donor through liver transplantation resulting in hypercholesterolemia in the recipient," American Journal of Transplantation, vol. 14, no. 12, pp. 2898-2902, 2014.

[8] J. Chen, X. Xu, J. Wu et al., "The stratifying value of hangzhou criteria in liver transplantation for hepatocellular carcinoma," PLoS ONE, vol. 9, no. 3, Article ID e93128, 2014.
[9] M. D. Stegall, G. Everson, G. Schroter, B. Bilir, F. Karrer, and I. Kam, "Metabolic complications after liver transplantation: diabetes, hypercholesterolemia, hypertension, and obesity," Transplantation, vol. 60, no. 9, pp. 1057-1060, 1995.

[10] S. Marubashi, K. Dono, K. Amano et al., "Steroid-free livingdonor liver transplantation in adults," Transplantation, vol. 80, no. 5, pp. 704-706, 2005.

[11] Q. Wei, X. Xu, C. Wang et al., "Efficacy and safety of a steroidfree immunosuppressive regimen after liver transplantation for hepatocellular carcinoma," Gut and Liver, vol. 10, no. 4, pp. 604$610,2016$. 


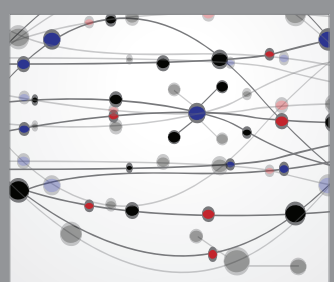

The Scientific World Journal
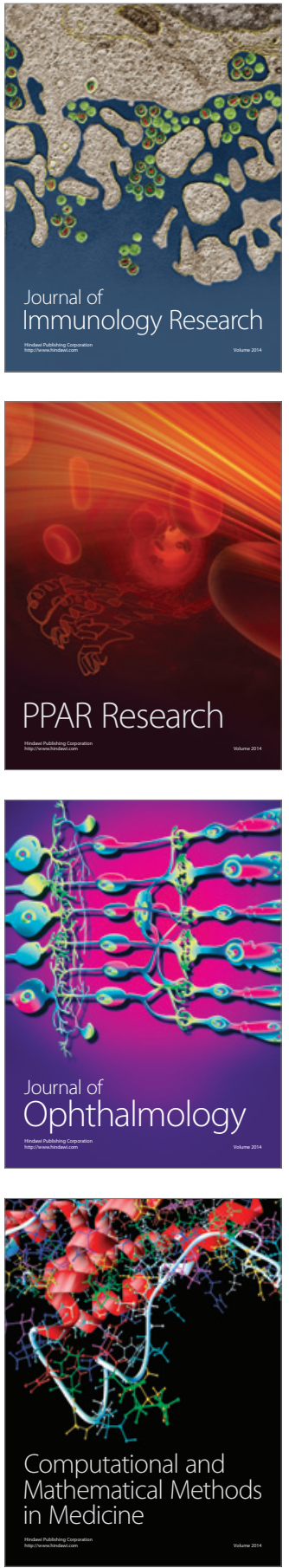

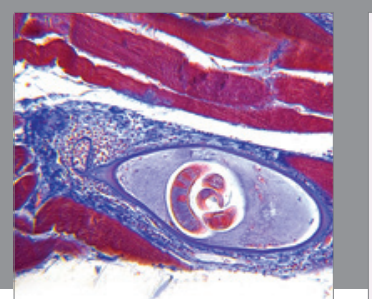

Gastroenterology Research and Practice
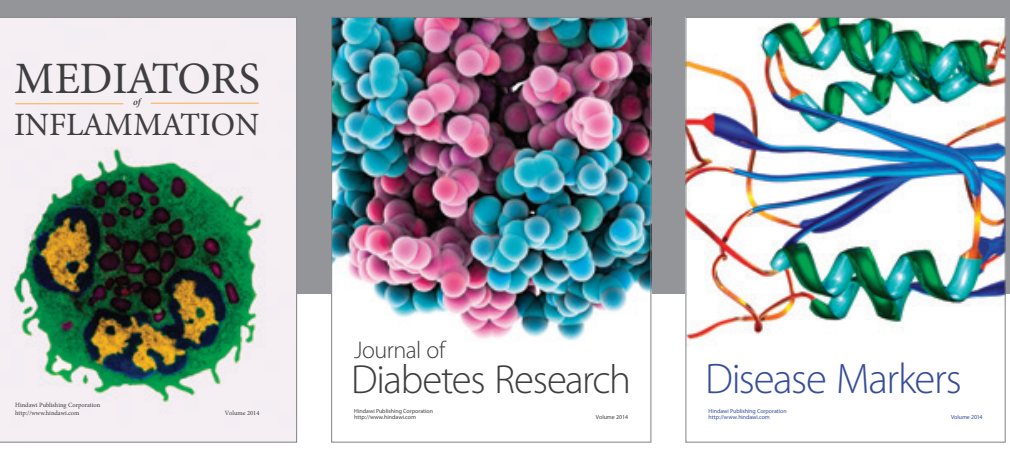

Disease Markers

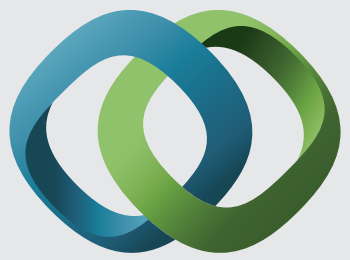

\section{Hindawi}

Submit your manuscripts at

https://www.hindawi.com
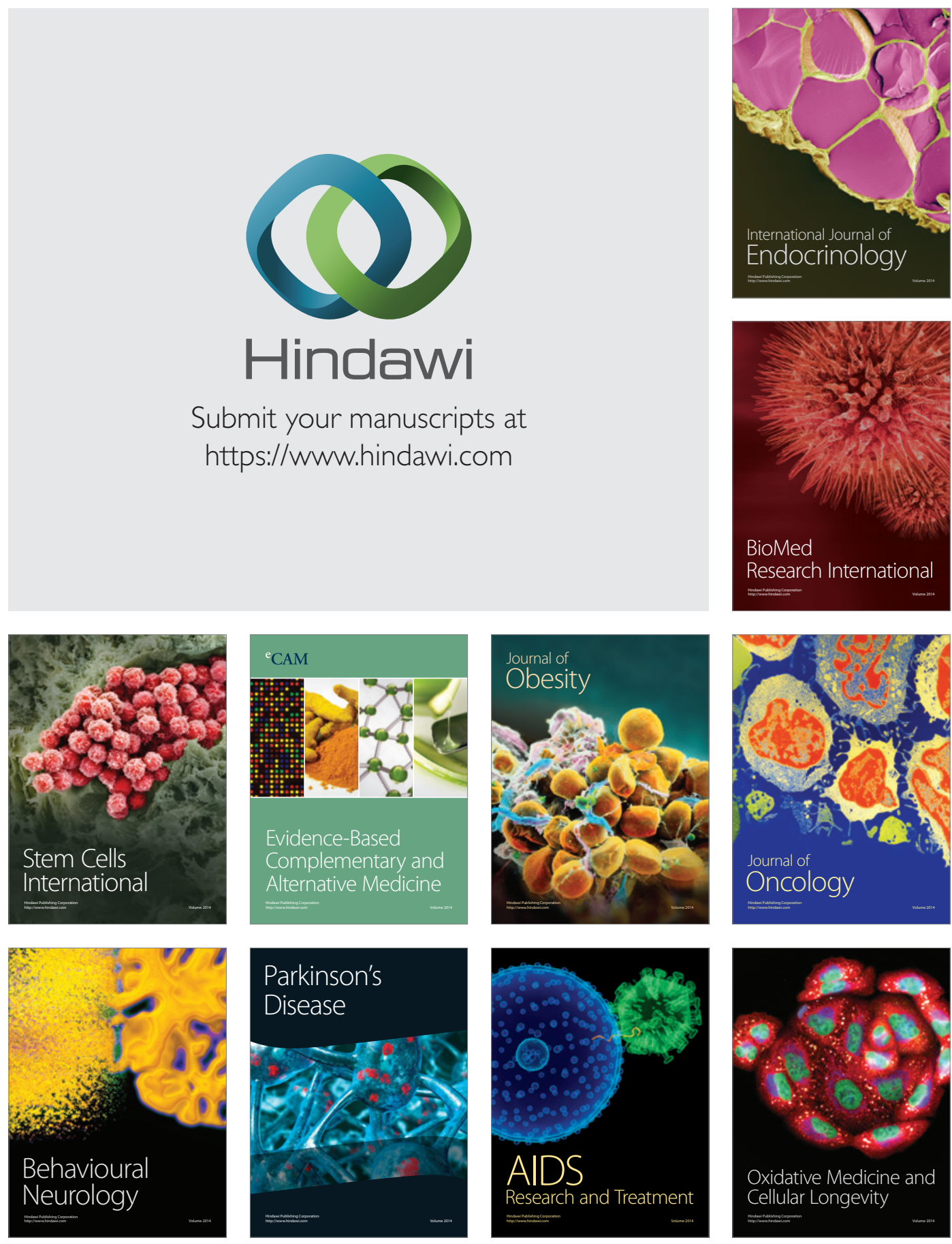